\title{
Pathological study of the 2019 novel coronavirus disease (COVID-19) through postmortem core biopsies
}

\author{
Sufang $\operatorname{Tian}^{1} \cdot$ Yong Xiong ${ }^{2} \cdot$ Huan $\mathrm{Liu}^{1} \cdot \mathrm{Li} \mathrm{Niu}^{1} \cdot$ Jianchun Guo ${ }^{1} \cdot$ Meiyan Liao ${ }^{3} \cdot$ Shu-Yuan Xiao $\mathbb{D}^{1,4}$
}

Received: 6 March 2020 / Revised: 23 March 2020 / Accepted: 23 March 2020 / Published online: 14 April 2020

(C) The Author(s), under exclusive licence to United States \& Canadian Academy of Pathology 2020

\begin{abstract}
Data on pathologic changes of the 2019 novel coronavirus disease (COVID-19) are scarce. To gain knowledge about the pathology that may contribute to disease progression and fatality, we performed postmortem needle core biopsies of lung, liver, and heart in four patients who died of COVID-19 pneumonia. The patients' ages ranged from 59 to 81 , including three males and one female. Each patient had at least one underlying disease, including immunocompromised status (chronic lymphocytic leukemia and renal transplantation) or other conditions (cirrhosis, hypertension, and diabetes). Time from disease onset to death ranged from 15 to 52 days. All patients had elevated white blood cell counts, with significant rise toward the end, and all had lymphocytopenia except for the patient with leukemia. Histologically, the main findings are in the lungs, including injury to the alveolar epithelial cells, hyaline membrane formation, and hyperplasia of type II pneumocytes, all components of diffuse alveolar damage. Consolidation by fibroblastic proliferation with extracellular matrix and fibrin forming clusters in airspaces is evident. In one patient, the consolidation consists of abundant intra-alveolar neutrophilic infiltration, consistent with superimposed bacterial bronchopneumonia. The liver exhibits mild lobular infiltration by small lymphocytes, and centrilobular sinusoidal dilation. Patchy necrosis is also seen. The heart shows only focal mild fibrosis and mild myocardial hypertrophy, changes likely related to the underlying conditions. In conclusion, the postmortem examinations show advanced diffuse alveolar damage, as well as superimposed bacterial pneumonia in some patients. Changes in the liver and heart are likely secondary or related to the underlying diseases.
\end{abstract}

\section{Introduction}

The coronavirus disease-19 (COVID-19), also known as novel coronavirus pneumonia, first occurred in Wuhan, China in early December 2019. Within 2 months, it had spread all over the world and caused 77,059 confirmed

Meiyan Liao

liaomy@whu.edu.cn

$\triangle$ Shu-Yuan Xiao

syxiao@uchicago.edu

1 Department of Pathology, Zhongnan Hospital of Wuhan University, Wuhan, China

2 Department of Infectious Diseases, Zhongnan Hospital of Wuhan University, Wuhan, China

3 Department of Radiology, Zhongnan Hospital of Wuhan University, Wuhan, China

4 Department of Pathology, University of Chicago Medicine, Chicago, IL 60637, USA infections, with 2446 fatal cases, in China by 24 February 2020 [1, 2]. The SARS-COV-2 (severe acute respiratory syndrome coronavirus 2) pathogen shares similarities with SARS-CoV in its genome sequence, biological behavior, and clinical manifestations [2-5]. It has four major structural proteins: the spike surface glycoprotein, small envelope protein, matrix protein, and nucleocapsid protein $[3,6]$. The spike protein binds to host receptors via the receptor-binding domains (RBDs) of angiotensinconverting enzyme 2 (ACE2) [7]. The ACE2 protein has been identified in various human organs, including the respiratory system, GI tract, lymph nodes, thymus, bone marrow, spleen, liver, kidney, and brain [8]. The common clinical manifestations of COVID-19 include fever, dry cough, dyspnea, muscle pain, confusion, headache, sore throat, rhinorrhea, chest pain, diarrhea, nausea, and vomiting [5]. SARS-COV-2 is believed to be more transmissible than SARS-COV [9].

Many studies on COVID-19 epidemiology and clinical characteristics have been published [2, 3, 10-12], but pathological data for this disease is still scarce. We 
reported on the pulmonary pathology of early-phase COVID-19 in two patients undergoing lung lobectomies for adenocarcinoma [13]. Others published a case report using postmortem biopsies [12]. So far, however, histopathologic data based on routine autopsies are still lacking. In the current study, we include postmortem biopsies of four patients from our hospital. The histopathologic findings are reported here.

\section{Materials and methods}

\section{Patients and clinical data}

All cases were from Zhongnan Hospital of Wuhan University and met the clinical diagnostic criteria provided by the National Health Commission of China (http://www.nhc.gov.cn/yzygj/ s7652m/202002/e84bd30142ab4d8982326326e4db22ea.shtml), as well as a positive nucleic acid test on nasopharyngeal swabs. Their electronic medical records were retrospectively reviewed to identify the patients' clinical features and laboratory findings. Demographic data, medical history, computed tomographic (CT) scans or X-ray images of the chest, laboratory findings (including nucleic acid tests, complete blood count (CBC), and other biochemical parameters of the liver and heart), and the duration of illness were all reviewed. The characteristics of all included cases are listed in Table 1. Dynamic CBC profiles, as well as chest CT images and X-rays, are shown in Figs. 1 and 2, respectively.

\section{Procurement of specimens and pathologic examination}

With permission from the patients' families, postmortem needle core biopsies were performed on visceral organs including the lungs, liver, and heart within an hour after death in a negative air isolation ward. The procedures were performed without ultrasound guidance, but the patients' last radiographic images and surface anatomic landmarks were used as references. The tissues were received fixed in neutral buffered formalin for over $24 \mathrm{~h}$, and then routinely processed under standard biosafety measures. Hematoxylin and eosin-stained sections were prepared and slides were examined by two pathologists (SFT and SYX). Immunohistochemistry (IHC) staining was performed in a liver specimen from Case 1, who had a history of CLL. It was used to verify subsets of the small lymphocytes found in portal tracts, using antibodies against CD20, CD3, CD5, CD23, CD4, and CD8 (Agilent Technologies, US). All antibodies were used in prediluted form, and IHC was performed using the automated Leica Bond-Max instrument.

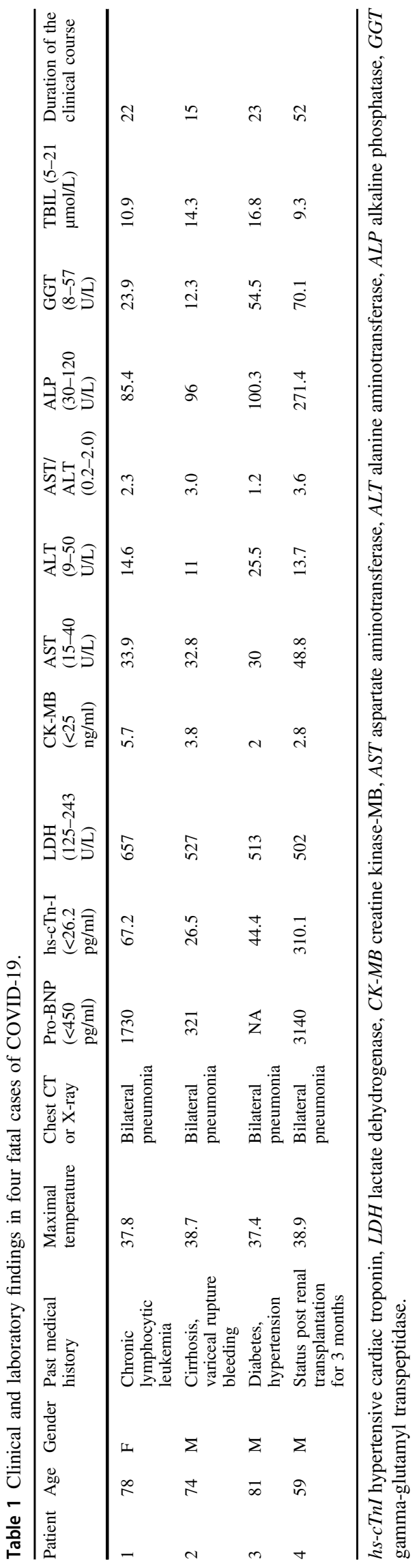




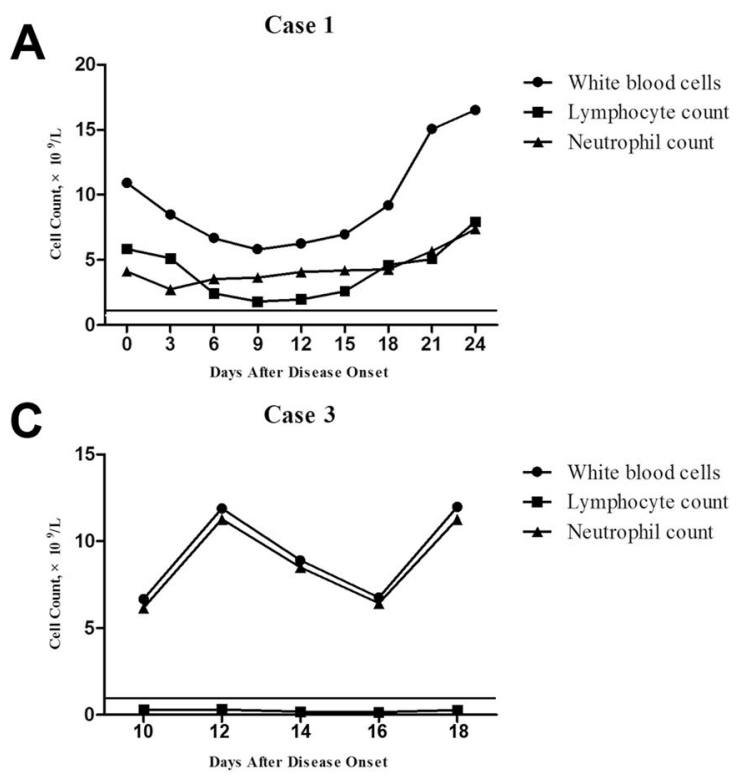

Fig. 1 Timeline charts of $\mathrm{CBC}$ including $\mathrm{WBC}$, neutrophil, and lymphocyte counts for four cases. The solid flat-line demarcates the lower normal limit of lymphocyte count $\left(1.1 \times 10^{9} / \mathrm{L}\right)$. In Case $1(\mathbf{a})$

\section{Real-time reverse transcription polymerase chain reaction assay for SARS-COV-2 in tissue}

Formalin-fixed, paraffin-embedded (FFPE) tissue blocks were used to prepare 20 serial sections of $4-\mu \mathrm{m}$ thick blocks. Total RNA was extracted using a sample RNA isolation kit (Catalog No.8.0224101X036G, Version B2.8, from Amoy Diagnostics Co. Ltd, Xiamen, China) and checked for concentration with the SMA4000 ultraviolet protein-nucleic acid microanalyzer. A real-time reverse transcriptase polymerase chain reaction (real-time RT-PCR) assay was run on the Mx3000P qPCR system with a 2019-nCoV nucleic acid detection kit (Catalog 8.0131901X024E, Version B1.0, from Amoy Diagnostics Co. Ltd, Xiamen, China) according to the manufacturer's protocol. Two target genes, the open reading frame1ab (ORF1ab) and nucleocapsid protein $(\mathrm{N})$ genes, were simultaneously amplified and monitored during the real-time RT-PCR assay. The primers for target 1 (ORF1ab) were forward $5^{\prime}$-CCCTGTGGGTTTTACACTTAA-3' and reverse $5^{\prime}$-ACGATTGTGCATCAGCTGA-3'; and the probe was $5^{\prime}$-ROX-CCGTCTGCGGTATGT-MGB-3'. The primers for target $2(\mathrm{~N})$ were forward $5^{\prime}$-GGGGAACTTC TCCTGCTAGAAT-3' and reverse 5'-CAGACATTTTGC TCTCAAGCTG- $3^{\prime}$; and the probe was $5^{\prime}$-FAM-CTGCTG CTTGACAGAT-MGB- $3^{\prime}$. A cycle threshold $(\mathrm{Ct})$ value less than 37 was defined as a positive, and a $\mathrm{Ct}$ value of 40 or more was defined as a negative. These testing criteria were based on recommendations by the National Institute for Viral Disease Control and Prevention (China) (http://ivdc. chinacdc.cn/kyjz/202001/t20200121_211337. html). Positive and negative controls were included. An internal control
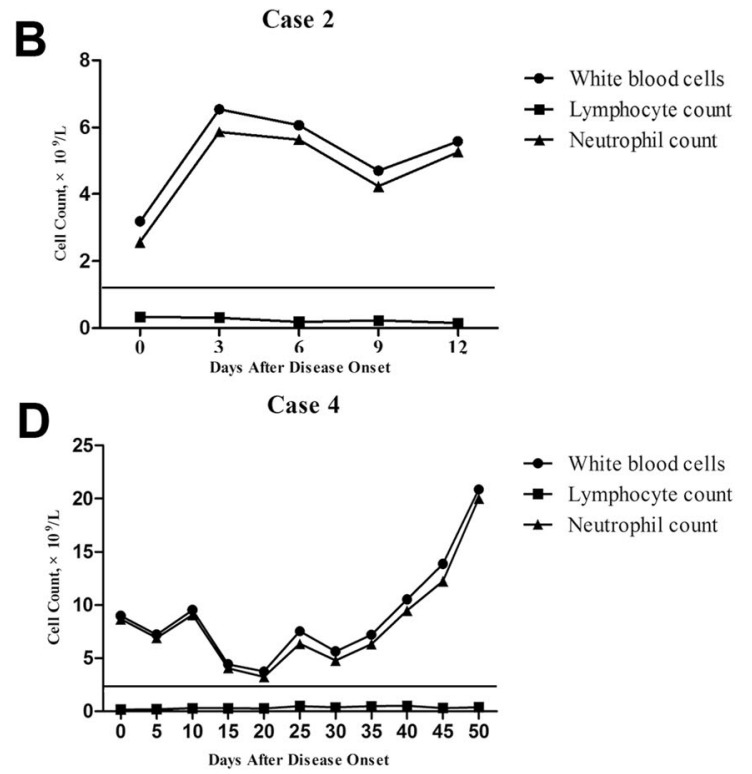

the elevated lymphocyte count is consistent with her history of chronic lymphocytic leukemia (CLL). Marked lymphocytopenia is seen in Cases 2, 3, and 4 (b, c, d).

HEX, corresponding to the house-keeping gene GUSB, which codes for beta-glucuronidase ( $\beta$-glucuronidase), was also included.

\section{Results}

\section{Clinical features and laboratory findings}

All patients had fever, with maximal temperatures reaching $38.9^{\circ} \mathrm{C}$. Nucleic acid tests on nasopharyngeal swabs were positive in all patients, some of whom underwent multiple tests during their hospital stay.

As shown in Table 1, the patients ranged in age from 59 to 81 years, and three out of four were male. They each had at least one underlying disease, such as chronic lymphocytic leukemia (CLL), cirrhosis, hypertension, diabetes, and renal transplantation. Although they were given comprehensive treatment, including intravenous antibiotics, antiviral therapy, and assisted oxygenation; specific treatment for their underlying diseases; as well as supportive treatment, their conditions deteriorated progressively toward death. Duration of the clinical course from onset of COVID-19 to death ranged from 15 to 52 days.

$\mathrm{CBC}$ over time are shown in Fig. 1, including white blood cell (WBC), neutrophil, and lymphocyte counts. Each patient had a unique profile, with the most prominent abnormality being the marked lymphocytopenia in Cases 2, 3, and 4. Case 1 had an elevated lymphocyte differential, consistent with her history of CLL. WBC and neutrophil counts varied in different patients. WBC counts were high 


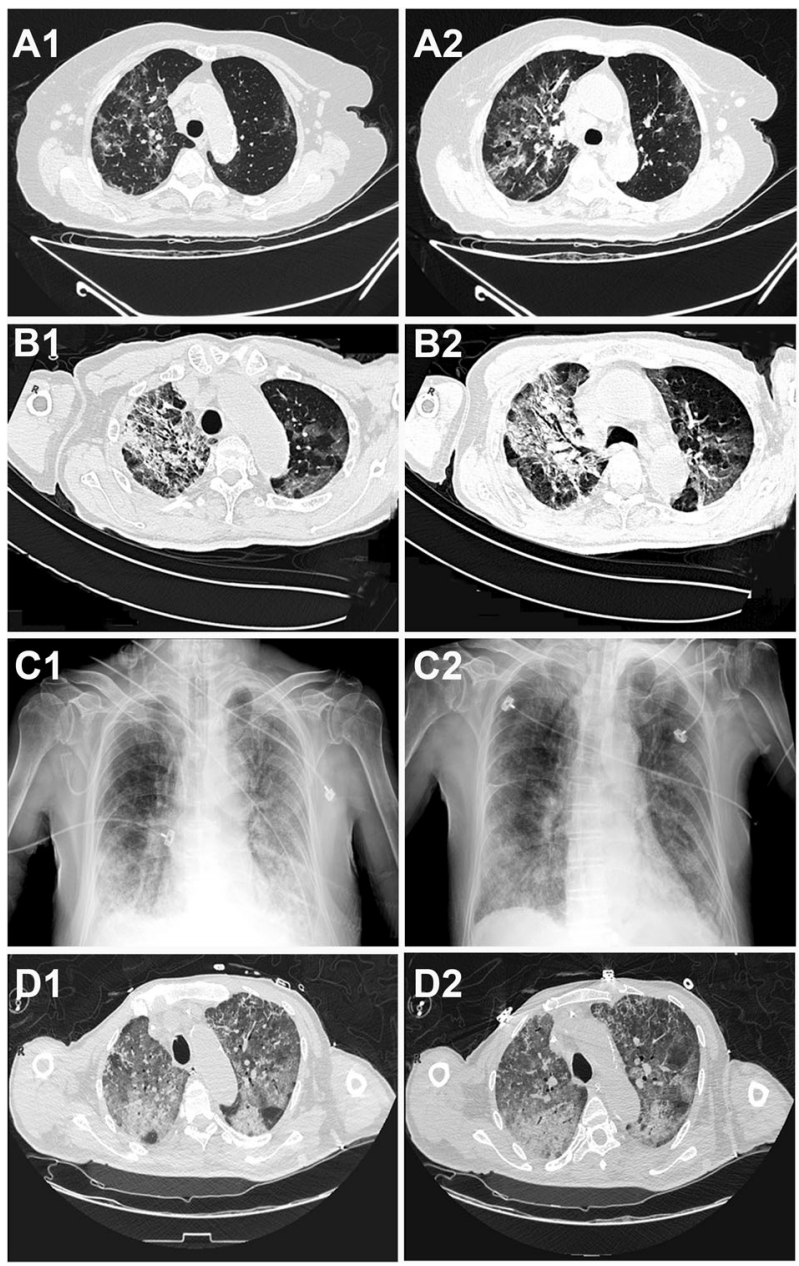

Fig. 2 Radiographic images of chest CT scan and X-ray from the four patients. For each patient, the left and right images represent an earlier and latter time-point, respectively. Chest CT from Case 1 showed multiple patchy GGO in the bilateral upper lobes of the lungs and appeared more prominent in the right upper lobe (RUL) (A1). Repeat CT showed similar changes as A1 but with thickened fascicles of vasculatures and bronchi (A2). In Case 2, patchy GGO, consolidation, and air bronchogram can be seen in RUL; scattered GGO can be identified in the left upper lobe (LUL) (B1). Repeat CT showed additional consolidation in LUL (B2). In Case 3, X-ray showed patchy high-density shadows in both lungs, which were more prominent in the lower lobes $(\mathrm{C} 1)$ and worsened during the couple of days before death (C2). In Case 4, diffuse GGO is seen in both lungs as well as consolidation in the posterior segment (D1), and additional air bronchogram can be detected in the later radiography (D2).

in Cases 1 and 3, and low in Case 2. Case 4 had a longer clinical course than the others, 52 days. His WBC was lowered as low as $2.79 \times 10^{9} \mathrm{~L}^{-1}$. However, in the end, all patients ultimately showed an elevated WBC.

Other clinical laboratory parameters are shown in Table 1, some corresponding to specific organ injuries to the liver and heart. For example, the elevation of pro-BNP and hypertensive cardiac troponin (hs-cTnI) in Case 1 suggests that this patient experienced myocardial injury. LDH increased in all patients. As for liver function tests, aspartate aminotransferase (AST), alanine aminotransferase (ALT), alkaline phosphatase (ALP), gamma-glutamyl transpeptidase (GGT), and total bilirubin were all essentially normal in this group of patients.

\section{Radiographic findings}

Chest CT scans and X-ray images are shown in Fig. 2. All patients had bilateral pneumonia with ground-glass opacity (GGO), with or without initial consolidations. More prominent consolidation appeared over time, especially in radiography taken before death. For instance, in Case 3, chest X-ray revealed patchy high-density opacity in both lungs, which was more prominent in the lower lobes (Fig. 2 $\mathrm{C} 1)$. This worsened during the last few days before death (Fig. 2 C2).

\section{Histologic findings}

Microscopic changes in the lungs varied among the four cases, but they were all consistent with diffuse alveolar damage (DAD). Cases 1, 2, and 3 showed hyaline membrane formation and vascular congestion, suggesting an acute-phase component (Fig. 3a-c). Occasionally, these were mixed with scant inflammatory cells. In addition, Case 1 exhibited pneumocyte injury with focal sloughing and formation of syncytial giant cells (Fig. 3a). There was also focal lymphocytic infiltration that likely represented her history of CLL. Case 2 exhibited no prominent inflammatory cellular infiltration (Fig. 3b), and Case 3 exhibited focal interstitial thickening (Fig. 3c). Case 4 showed more advanced changes. In addition to remnants of hyaline membranes in some airspaces, large areas of intra-alveolar hemorrhages and intra-alveolar fibrin cluster formation were observed nearby (Fig. 3d). In addition, the alveolar wall contained increased stromal cells, fibrin, and infiltration by mononuclear inflammatory cells. Together with type II pneumocyte hyperplasia, these led to interstitial thickening (Fig. 3e). Fibrinoid necrosis of the small vessels was noted as well (Fig. 3e, inset). In this patient, there was also evidence of consolidation by abundant intra-alveolar neutrophilic infiltration, consistent with bronchopneumonia of a superimposed bacterial infection (Fig. 3f).

The liver sections only showed mild sinusoidal dilatation, a common nonspecific change in terminally ill hospitalized patients. Nuclear glycogen accumulation in hepatocytes, focal macrovesicular steatosis, and dense atypical small lymphocytes in portal tracts were seen in Case 1, consistent with her medical history of CLL (Fig. 4a). Liver tissue from Case 2 contained regenerative nodules and thick fibrous bands, consistent with his history of cirrhosis (Fig. 4b). In addition to mild zone 3 sinusoidal dilatation 


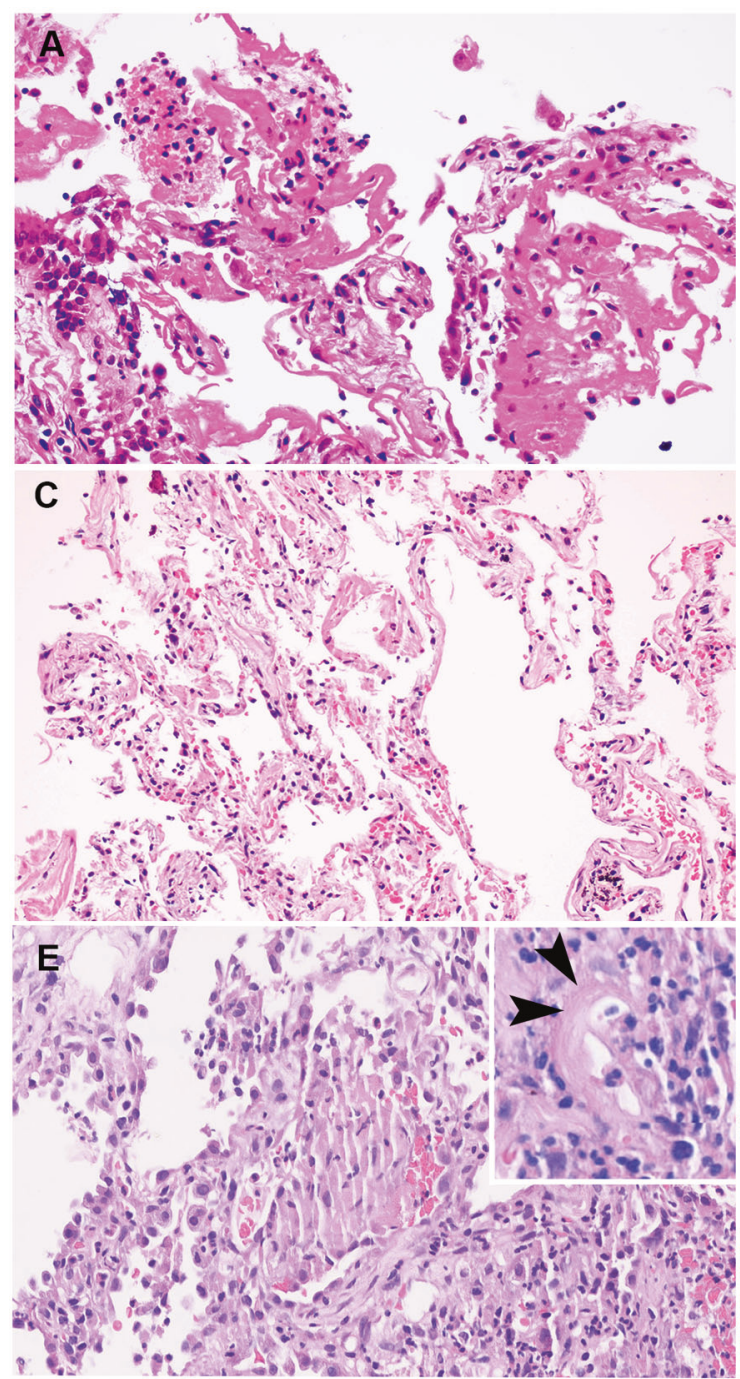

Fig. 3 Histologic changes in the lungs. a Case 1: thick hyaline membrane mixed with desquamative pneumocytes and mononuclear inflammatory cells. b Case 2: more delicate hyaline membranes without evident inflammatory infiltration. c Case 3: focal hyaline membrane, type II pneumocyte hyperplasia, and mild interstitial thickening. d Case 4: alveolar spaces were filled with red blood cell

(Fig. 4c), mild lobular lymphocytic infiltration was also noted in Cases 3 and 4 (Fig. 4d). In general, there was no significant lymphocytic infiltration of the portal tracts. Case 4 also showed patchy hepatic necrosis in the periportal and centrilobular areas (Fig. 4e, f). No obvious fatty changes were seen in Case 2, 3, or 4 .

Heart biopsies were only obtained from Cases 1 and 4 . Their endocardia and myocardia did not contain inflammatory cellular infiltration. Although, focally, the myocardium appeared irregular in shape with darkened cytoplasm, these changes were not sufficient for interpretation as acute myocardial injury. There were various degrees of focal edema, interstitial fibrosis, and myocardial hypertrophy. These latter

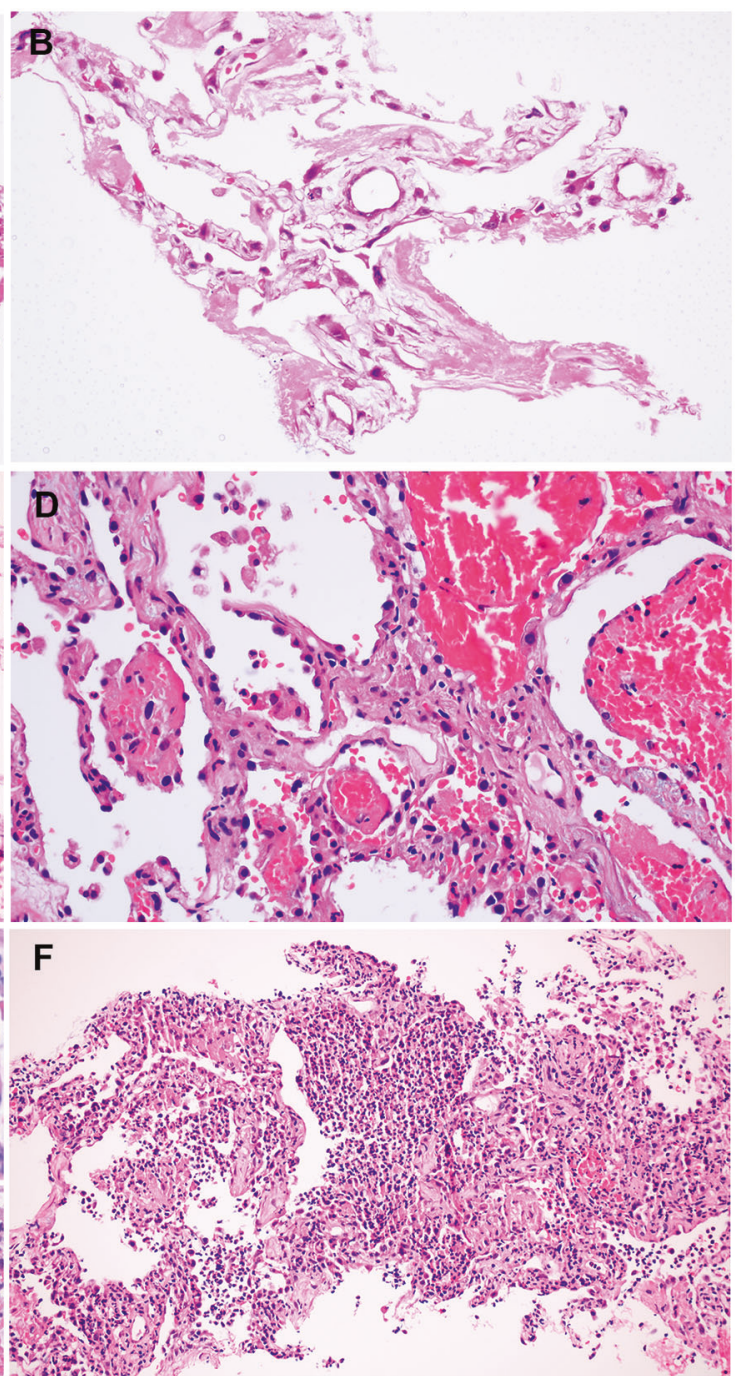

exudation, and small fibrin plugs seen in adjacent alveoli. e Organization with intra-alveolar fibroblasts mixed with fibrin and inflammatory cellular infiltration. Diffuse type II pneumocyte hyperplasia in the background (inset: fibrinoid vascular necrosis, arrow heads). f Changes of bronchopneumonia with prominent neutrophilic infiltration filling up alveolar spaces.

findings likely represent pre-existing changes related to patients' underlying diseases, such as hypertension-associated myocardial hypertrophy and past ischemic injury.

The key histological findings are summarized in Table 2 .

\section{RT-PCR assay for SARS-COV-2 in tissue}

The RT-PCR assay for SARS-COV-2 was performed on selected tissue blocks. Heart and liver tissues from Case 1 and lung tissue from Case 2 were positive. RT-PCR was not performed on lung tissue from Case 1 due to insufficient remaining tissue. Tests on liver tissue from Cases 3 and 4 and heart from Case 4 were negative. 

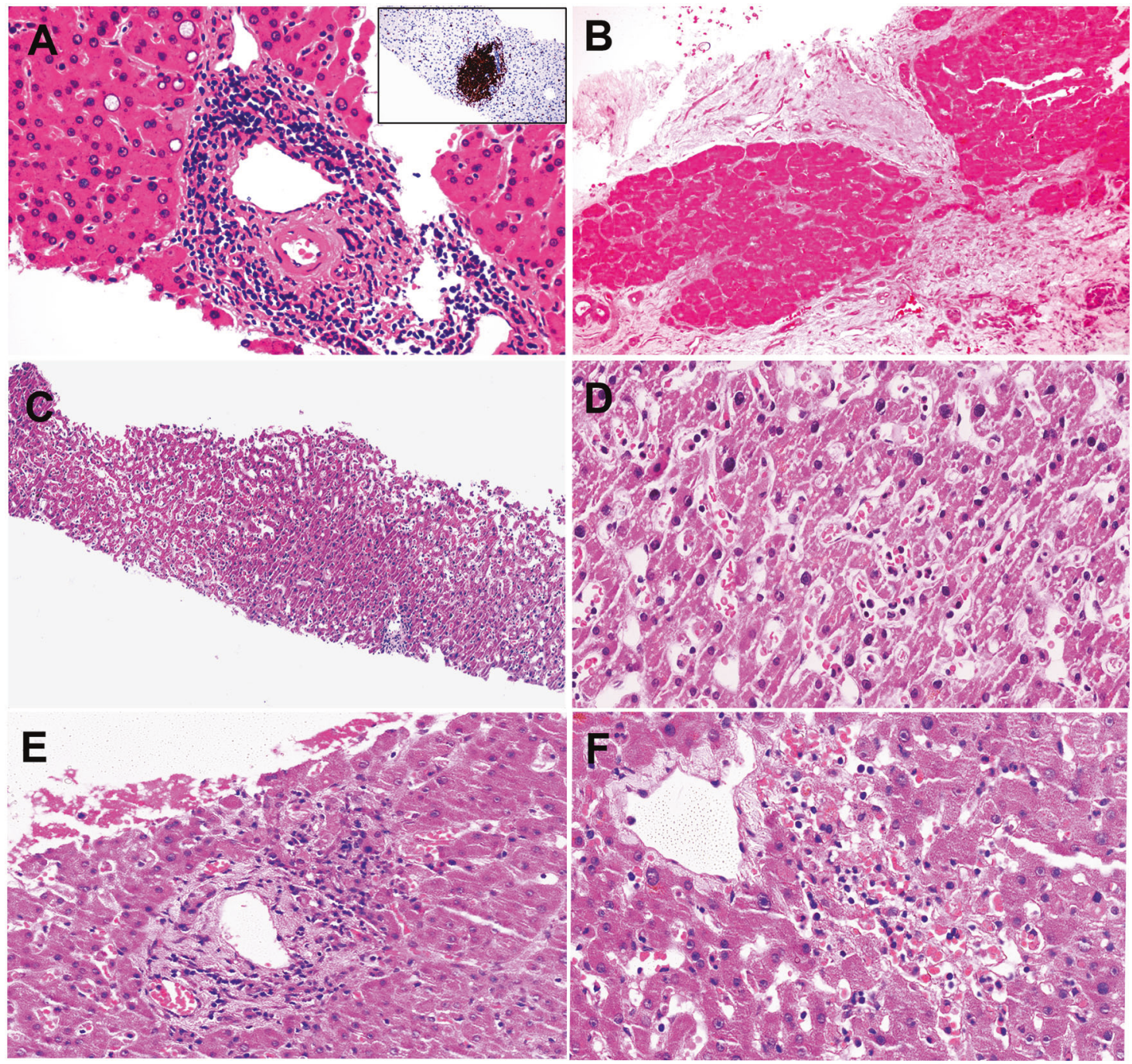

Fig. 4 Pathological findings in liver from all four cases. a Dense portal infiltration by atypical small lymphocytes (insert: CD20 immunostaining), and focal glycogenated nuclei in hepatocyte in Case 1. b Cirrhotic nodules with thick fibrosis in Case 2. c Mild sinusoidal dilatation with increased lymphocytic infiltration. d Higher power view showing sinusoidal lymphocytes. e Focal hepatic necrosis in periportal zone. f Focal centrilobular hepatic necrosis in Case 4.

\section{Discussion}

To a certain extent, the clinical characteristics of the four patients in this study are representative of the fatal cases of COVID-19 as described in a previous study from our hospital [11]. Their blood biochemical parameters are similar to those described in the literature $[5,11]$.

The main pathologic findings from the lungs of these fatal cases of COVID-19 pneumonia include hyaline membrane formation, fibrin exudates, epithelial damage, and diffuse type II pneumocyte hyperplasia, which are all features of DAD. Mild thickening of alveolar walls is also evident in some cases, suggesting a more advanced stage. However, mature fibrosis is not seen. A common feature of the chest radiological findings of COVID-19 pneumonia is
GGO, which may represent an early exudative phase of COVID-19 pneumonia. With advancing disease, consolidation occurs in severely ill patients, likely due to intraalveolar organization by fibroblastic proliferation with extracellular matrix formation, and interstitial thickening. In some patients, the radiographic consolidation may be caused by massive intra-alveolar neutrophilic infiltration, likely due to superimposed bacterial pneumonia. These were identified in Case 4, who had a longer clinical course of 52 days. Previously, we identified pathological changes in resected lung specimens of two pre-symptomatic COVID-19 patients, including edema, proteinaceous exudate, and focal hyperplasia of pneumocytes with only patchy inflammatory cellular infiltration, and the absence of hyaline membranes [13]. These evidently represent an early 


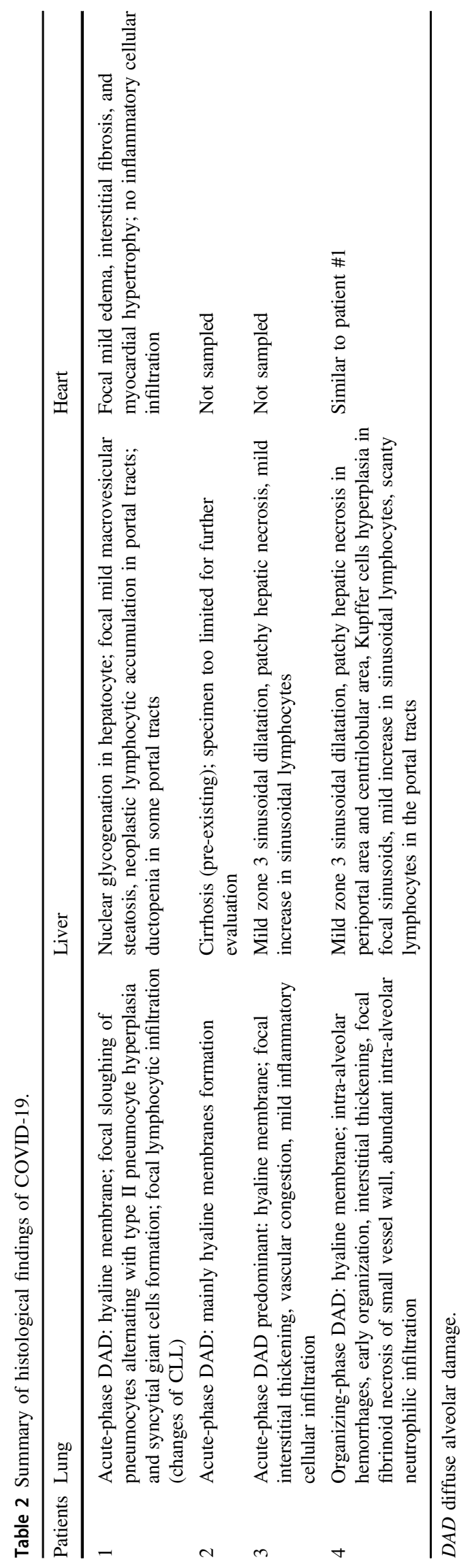

phase of COVID-19 pneumonia and are consistent with GGO in chest CT scans.

Recent studies have shown that the novel coronavirus SARS-COV-2 binds to ACE2 receptors on target cells through the RBD on viral particles, leading to replication [7]. ACE2 is localized in various human tissues including the liver [8]. Several clinical studies have shown biochemical evidence of liver injuries [14, 15]. However, only limited histologic hepatic abnormalities are noted in our patients. It has been shown that bile duct epithelium expresses higher density of ACE2 [14]. Since these needle biopsies were limited in sampling, not enough portal tracts were included for thorough examination. The mild lobular lymphocytic infiltration is likely related to the viral infection itself.

To our surprise, no significant histologic changes or inflammation were identified in the myocardia of two cases examined. Clinically many patients with COVID19 showed biochemical parameters consistent with myocardial injury. We suspect that acute tissue injuries related to lowered blood oxygen saturation are too mild to cause visible morphologic changes. However, it may be premature to say whether or not there are viral-induced pathologic changes to the myocardium. Additional cases should be studied.

Excluding the patient with underlying CLL, there is uniform lymphocytopenia in these cases. Some investigators suggested that this might be due to redistribution of lymphocytes to affected organ tissues. However, this phenomenon is not observed in our study. The mild increase in sinusoidal lymphocytes in the liver is not sufficient to account for the lymphocytopenia. Another possibility may be virus-related suppression of bone marrow, as described in SARS-COV $[8,16]$. Unfortunately, no bone marrow tissue has been studied so far.

Pathologically, COVID-19 pneumonia shows overlapping features with SARS. Both exhibit fluid exudation, vascular congestion, inflammatory cellular infiltration, and hyaline membrane formation. With a clinical course of 10 days or shorter, SARS is characterized by acute exudation and hyaline membrane formation. Squamous metaplasia, multinucleated giant cells, fibrin plugs, and pulmonary infarct can be seen in some cases beyond 14 days [17, 18]. In SARS cases lasting longer than 20 days, interstitial and alveolar fibroblast proliferation, septal thickening, and organizing pneumonia are seen. Reported pathologic changes for SARS come mostly from full autopsy studies, which should be more representative of the full picture, but they may also be complicated by postmortem changes and other superimposed changes of the terminally ill. In contrast, specimens included in the current study were obtained $1 \mathrm{~h}$ after death, largely avoided postmortem degenerative changes. Our previous study of early pathologic changes of COVID-19 pneumonia in two patients 
showed relatively simple, early exudative phase changes, without hyaline membrane formation, or significant inflammatory cellular infiltration. More advanced pathologic abnormalities are seen in the current study of fatal cases.

In China, medical autopsies are not commonly performed. In addition, there are special biosafety concerns associated with patients who have died of COVID-19. Therefore, no autopsies could be performed on patients who died of COVID-19 in the early phase of the outbreak. Autopsies were later permitted to be performed under stringent biosafety regulations, but in Wuhan, there is a shortage of autopsy labs meeting these requirements. Faced with these challenges, we sought for alternatives such as the postmortem biopsies used in this study. This method was also used by another team from Beijing at around the same time [12]. We recognize that due to limited sampling in needle core procedures, studies using postmortem biopsies carry certain limitations. For example, RT-PCT showed direct evidence of the viral sequence in the liver in only one of three cases tested. This may be due to either limited test sensitivity or sampling problem. Because Wuhan is still under lockdown, technical services remain very limited. Rather than working up a protocol optimized for FFPE tissue, a PCR kit designed for pharyngeal swabs was used instead. Second, there is a possibility of RNA degradation, which is common in clinical samples, particularly when specimens are not immediately stored in a transfer medium suitable for preserving RNA. Hopefully, future studies will be able to avoid these challenges and provide a more complete picture of the viral distribution in different tissues.

In this study, we have found that the pathologic basis for the COVID-19 pneumonia are advanced DAD and superimposed bacterial pneumonia in some patients. Early organization is noted but without marked fibrosis. Changes in the liver and heart are limited or related to the underlying diseases. To further understand the pathogenesis of COVID-19, studies including more patients with different ages and physiological backgrounds are needed. In addition, proper animal models need to be established, mimicking not only the infection itself but also the pattern of disease progression in humans.

Acknowledgements We thank Dr Alya Husain of the University of Chicago Medicine for her expert consultation on the histopathology of the lung and heart.

\section{Compliance with ethical standards}

Conflict of interest The authors declare that they have no conflict of interest.

Publisher's note Springer Nature remains neutral with regard to jurisdictional claims in published maps and institutional affiliations.

\section{References}

1. Chan JF, Yuan S, Kok KH, To KK, Chu H, Yang J, et al. A familial cluster of pneumonia associated with the 2019 novel coronavirus indicating person-to-person transmission: a study of a family cluster. Lancet. 2020;395:514-23.

2. Zhu N, Zhang D, Wang W, Li X, Yang B, Song J, et al. A novel coronavirus from patients with pneumonia in China, 2019. N Engl J Med. 2020;382:727-33.

3. Wu A, Peng Y, Huang B, Ding X, Wang X, Niu P, et al. Genome composition and divergence of the novel coronavirus (2019-nCoV) originating in China. Cell Host Microbe. 2020; 27:325-8.

4. Li F. Structure, function, and evolution of coronavirus spike proteins. Annu Rev Virol. 2016;3:237-61.

5. Chen N, Zhou M, Dong X, Qu J, Gong F, Han Y, et al. Epidemiological and clinical characteristics of 99 cases of 2019 novel coronavirus pneumonia in Wuhan, China: a descriptive study. Lancet. 2020;395:507-13.

6. Ge XY, Li JL, Yang XL, Chmura AA, Zhu G, Epstein JH, et al. Isolation and characterization of a bat SARS-like coronavirus that uses the ACE2 receptor. Nature. 2013;503:535-8.

7. Lan J, G JW, Yu JF, Shan SS, Zhou H, Fan SL, et al. Crystal structure of the 2019-nCoV spike receptor-binding domain bound with the ACE2 receptor. 2020. https://www.biorxiv.org/content/ 10.1101/2020.02.19.956235v1.

8. Hamming I, Timens W, Bulthuis ML, Lely AT, Navis G, van Goor H. Tissue distribution of ACE2 protein, the functional receptor for SARS coronavirus. A first step in understanding SARS pathogenesis. J Pathol. 2004;203:631-7.

9. Wrapp D, Wang N, Corbett KS, Goldsmith JA, Hsieh CL, Abiona O, et al. Cryo-EM structure of the 2019-nCoV spike in the prefusion conformation. Science. 2020;367:1260-3.

10. Huang C, Wang Y, Li X, Ren L, Zhao J, Hu Y, et al. Clinical features of patients infected with 2019 novel coronavirus in Wuhan, China. Lancet. 2020;395:497-506.

11. Wang D, Hu B, Hu C, Zhu F, Liu X, Zhang J, et al. Clinical characteristics of 138 hospitalized patients with 2019 novel coronavirus-infected pneumonia in Wuhan, China. JAMA. 2020; 323:1061-9.

12. Xu Z, Shi L, Wang Y, Zhang J, Huang L, Zhang C, et al. Pathological findings of COVID-19 associated with acute respiratory distress syndrome. Lancet Respir Med. 2020;8:420-2.

13. Tian S, Hu W, Niu L, Liu $\mathrm{H}, \mathrm{Xu} \mathrm{H}$, Xiao SY. Pulmonary pathology of early-phase 2019 novel coronavirus (COVID-19) pneumonia in two patients with lung cancer. J Thorac Oncol. https://doi.org/10.1016/j.jtho.2020.02.010. (in press)

14. Chai X, Hu L, Zhang Y, Han W, Lu Z, Ke A, Zhou J, et al. Specific ACE2 expression in cholangiocytes may cause liver damage after 2019-nCoV infection. 2020. https://www.biorxiv. org/content/10.1101/2020.02.03.931766v1.

15. Fan Z, Chen L, Li J,Tian C, Zhang Y, Huang S, et al. Clinical features of COVID-19 related liver damage. 2020. https://www. medrxiv.org/content/10.1101/2020.02.26.20026971v2.

16. Yang M, Hon KL, Li K, Fok TF, Li CK. The effect of SARS coronavirus on blood system: its clinical findings and the pathophysiologic hypothesis. Zhongguo Shi Yan Xue Ye Xue Za Zhi. 2003;11:217-21.

17. Franks TJ, Chong PY, Chui P, Galvin JR, Lourens RM, Reid $\mathrm{AH}$, et al. Lung pathology of severe acute respiratory syndrome (SARS): a study of 8 autopsy cases from Singapore. Hum Pathol. 2003;34:743-8.

18. Hwang DM, Chamberlain DW, Poutanen SM, Low DE, Asa SL, Butany J. Pulmonary pathology of severe acute respiratory syndrome in Toronto. Mod Pathol. 2005;18:1-10. 